\title{
Some Properties of Generalized Gegenbauer Matrix Polynomials
}

\author{
Ghazala Yasmin \\ Department of Mathematics, Aligarh Muslim University, Aligarh 202002, India \\ Correspondence should be addressed to Ghazala Yasmin; ghazala30@gmail.com
}

Received 24 December 2013; Accepted 9 May 2014; Published 29 May 2014

Academic Editor: Sivaguru Sritharan

Copyright (C) 2014 Ghazala Yasmin. This is an open access article distributed under the Creative Commons Attribution License, which permits unrestricted use, distribution, and reproduction in any medium, provided the original work is properly cited.

\begin{abstract}
Various new generalized forms of the Gegenbauer matrix polynomials are introduced using the integral representation method, which allows us to express them in terms of Hermite matrix polynomials. Certain properties for these new generalized Gegenbauer matrix polynomials such as recurrence relations and expansion in terms of Hermite matrix polynomials are derived. Further, several families of bilinear and bilateral generating matrix relations for these polynomials are established and their applications are presented.
\end{abstract}

\section{Introduction}

Theory of generalized and multivariable special functions has provided new means of analysis to deal with the majority of problems in mathematical physics which find broad practical applications. Further, an extension to the matrix framework of special functions is special matrix functions. The study of special matrix polynomials is important due to their applications in certain areas of statistics, physics, and engineering. In recent years, some results in the theory of classical orthogonal polynomials have been extended to orthogonal matrix polynomials [1], which forms an emergent field and plays an important role from both the theoretical and practical point of view. Orthogonal matrix polynomials appear in connection with representation theory, matrix expansion problems, prediction theory, and in the reconstruction of matrix functions. The Laguerre and Hermite matrix polynomials and their extension and generalizations have been introduced and studied in [2-9] for matrices in $\mathbb{C}^{N \times N}$ whose eigenvalues are all situated in right open halfplane.

If $D_{0}$ is the complex plane cut along the negative real axis and $\log (z)$ denotes the principal logarithm of $z$, then $z^{1 / 2}$ represents $\exp ((1 / 2) \log (z))$. If $A$ is a matrix in $\mathbb{C}^{N \times N}$ with $\sigma(A) \subset D_{0}$ where $\sigma(A)$ (the spectrum of $A$ ) is the set of all the eigenvalues of $A$, then $A^{1 / 2}=\sqrt{A}$ denotes the image by $z^{1 / 2}$ of the matrix functional calculus acting on the matrix $A$.
Throughout this paper, we assume that $A$ is a positive stable matrix in $\mathbb{C}^{N \times N}$; that is, $A$ satisfies the following condition:

$$
\operatorname{Re}(z)>0, \quad \text { for all } z \in \sigma(A) \text {. }
$$

First, we recall that the Chebyshev polynomials (CP) $U_{n}(x)$ and Gegenbauer polynomials $(\mathrm{GP}) C_{n}^{\mu}(x)$ are defined in $[10]$ as

$$
\begin{gathered}
U_{n}(x)=\sum_{k=0}^{[n / 2]} \frac{(-1)^{k}(n-k) !(2 x)^{n-2 k}}{(n-2 k) ! k !}, \\
C_{n}^{\mu}(x)=\frac{1}{\Gamma(\mu)} \sum_{k=0}^{[n / 2]} \frac{(-1)^{k} \Gamma(n+\mu-k)(2 x)^{n-2 k}}{(n-2 k) ! k !} .
\end{gathered}
$$

Next, we recall certain recently introduced Hermite matrix and Laguerre matrix polynomials. We mentioned these matrix polynomials in Table 1 .

Due to the importance of generalized Hermite matrix polynomials, which find broad practical applications recently, Batahan [2] introduces a matrix version of Chebyshev polynomials in terms of $2 \mathrm{VHMaP} H_{n}(x, y, A)$ (Table $1(\mathrm{I})$ ).

To give an idea of the procedure adopted in [11], we use $2 \mathrm{VHMaP} H_{n}(x, y, A)$ to introduce the generalized Chebyshev matrix polynomials of the second kind (gCMaP) 


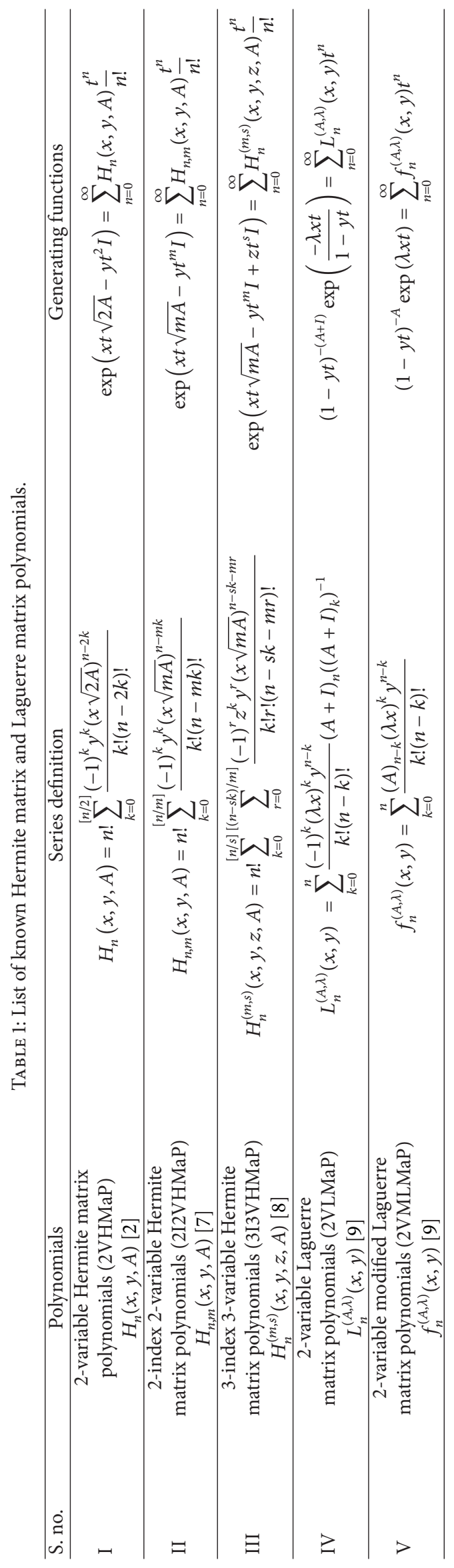


$U_{n}(x, y, A)$ by the following integral representation and series definitions [2, p.91]:

$$
\begin{gathered}
U_{n}(x, y, A)=\frac{1}{n !} \int_{0}^{\infty} e^{-t} t^{n} H_{n}\left(x, \frac{y}{t}, A\right) d t \\
U_{n}(x, y, A)=\sum_{k=0}^{[n / 2]} \frac{(-1)^{k}(n-k) ! y^{k}(x \sqrt{2 A})^{n-2 k}}{(n-2 k) ! k !} \quad(n \geq 0),
\end{gathered}
$$

respectively. The relevant generating function which was obtained by using the integral representation (4) is given as

$$
\left(I-x \xi \sqrt{2 A}+y \xi^{2} I\right)^{-1}=\sum_{n=0}^{\infty} U_{n}(x, y, A) \xi^{n} .
$$

It is evident that

$$
U_{n}(x, y, A)=y^{n / 2} U_{n}\left(\frac{x}{\sqrt{y}}, A\right) .
$$

Motivated by the work of Dattoli et al. [11] who have used the link between Hermite and Gegenbauer polynomials to introduce generalized forms of Gegenbauer polynomials where the strategy of generalization outlined in [11] benefits from the variety of existing Hermite polynomials in this paper, Hermite matrix polynomials and its various generalizations were exploited to introduce a matrix version of Gegenbauer polynomials.

The use of integral representations relating to Gegenbauer matrix polynomials and Hermite matrix polynomials is a fairly useful tool of analysis which also offers interesting criteria of generalizations. By combining the wealth of different forms of Hermite matrix polynomials and the flexibility of the proposed representations, we can establish within such a framework a systematic procedure of generalization involving new representations of Gegenbauer matrix and generalized Gegenbauer matrix polynomials. Further, certain properties involving newly introduced 3-variable 1-parameter generalized Gegenbauer matrix polynomials (3V1PgGeMaP) ${ }_{(m, s)} C_{n}^{(\mu)}(x, y, z ; \alpha, A)$ are derived which include recurrence relations, expansion in the series of Hermite matrix polynomial. The bilinear and bilateral generating matrix relations for $3 \mathrm{~V} 1 \mathrm{PgGeMaP}{ }_{(m, s)} C_{n}^{(\mu)}(x, y, z ; \alpha, A)$ are also established which further leads to certain new and known bilinear and bilateral generating matrix relations as special case.

In Section 2, we introduce two forms of Gegenbauer matrix polynomials. In Section 3, we introduce two forms of generalized Gegenbauer matrix polynomials and derive certain properties involving these polynomials. In Section 4, we obtain expansion for $3 \mathrm{~V} 1 \mathrm{PgGeMaP}{ }_{(m, s)} C_{n}^{(\mu)}(x, y, z ; \alpha, A)$. In Section 5, we establish certain bilinear and bilateral generating matrix relations involving 3V1PgGeMaP. In Section 6, concluding remarks are given.

\section{Gegenbauer Matrix Polynomials}

The 2VHMaP $H_{n}(x, y, A)$ (Table $1(\mathrm{I})$ ) will be exploited here to introduce a matrix version of Gegenbauer polynomials.
The Gegenbauer matrix polynomials (GeMaP) $C_{n}^{(\mu)}(x, A)$ involving $H_{n}(x, y, A)$ can be define in the following form:

$$
C_{n}^{(\mu)}(x, A)=\frac{1}{n ! \Gamma(\mu)} \int_{0}^{\infty} e^{-t} t^{n+\mu-1} H_{n}\left(x, \frac{1}{t}, A\right) d t .
$$

It is evident that in view of the relation that

$$
t^{n} H_{n}(x, y, A)=H_{n}\left(x t, y t^{2}, A\right) .
$$

Equation (8) can be expressed equivalently as

$$
C_{n}^{(\mu)}(x, A)=\frac{1}{n ! \Gamma(\mu)} \int_{0}^{\infty} e^{-t} t^{\mu-1} H_{n}(x t, t, A) d t .
$$

Now, making use of (2) and formula (see [12])

$$
a^{-\nu}=\frac{1}{\Gamma(\nu)} \int_{0}^{\infty} e^{-a t} t^{\nu-1} d t
$$

we find that the $\operatorname{GeMaP} C_{n}^{(\mu)}(x, A)$ are defined by the following series:

$$
C_{n}^{(\mu)}(x, A)=\frac{1}{\Gamma(\mu)} \sum_{k=0}^{[n / 2]} \frac{(-1)^{k} \Gamma(n-k+\mu)(x \sqrt{2 A})^{n-2 k}}{(n-2 k) ! k !} .
$$
find

Multiplying (10) by $\xi^{n}$ and then summing up over $n$, we

$$
\sum_{n=0}^{\infty} C_{n}^{(\mu)}(x, A) \xi^{n}=\frac{1}{\Gamma(\mu)} \int_{0}^{\infty} e^{-t} t^{\mu-1} \sum_{n=0}^{\infty} H_{n}(x t, t, A) \frac{\xi^{n}}{n !} d t
$$

Now, using the generating function of $H_{n}(x, y, A)$ (Table $1(\mathrm{I})$ ) in the r.h.s. of the above equation and then using relation (11) on the resultant equation, we get the following generating function of the $\operatorname{GeMaP}_{n}^{(\mu)}(x, A)$ :

$$
\sum_{n=0}^{\infty} C_{n}^{(\mu)}(x, A) \xi^{n}=\left(I-x \xi \sqrt{2 A}+\xi^{2} I\right)^{-\mu} \quad(\mu \neq 0) .
$$

Further, generalization of $C_{n}^{(\mu)}(x, A)$ can be obtained by introducing the 2-variable 1-parameter Gegenbauer matrix polynomials (2V1PGeMaP) $C_{n}^{(\mu)}(x, y ; \alpha, A)$ by using $2 \mathrm{VHMaP} H_{n}(x, y, A)$ (Table $\left.1(\mathrm{I})\right)$ in the following form:

$$
C_{n}^{(\mu)}(x, y ; \alpha, A)=\frac{1}{n ! \Gamma(\mu)} \int_{0}^{\infty} e^{-\alpha t} t^{n+\mu-1} H_{n}\left(x, \frac{y}{t}, A\right) d t
$$

Also, in view of relation (9), the above equation can be expressed equivalently as

$$
C_{n}^{(\mu)}(x, y ; \alpha, A)=\frac{1}{n ! \Gamma(\mu)} \int_{0}^{\infty} e^{-\alpha t} t^{\mu-1} H_{n}(x t, y t, A) d t .
$$


By employing the same procedure as above, we can easily obtain the series definition and generating function for the $2 \mathrm{~V} 1 \mathrm{PGeMaP} C_{n}^{(\mu)}(x, y ; \alpha, A)$ as

$$
\begin{gathered}
C_{n}^{(\mu)}(x, y ; \alpha, A) \\
=\frac{1}{\Gamma(\mu)} \sum_{k=0}^{[n / 2]} \frac{(-y)^{k} \Gamma(n-k+\mu)(x \sqrt{2 A})^{n-2 k}}{(n-2 k) ! k ! \alpha^{n-k+\mu}}, \\
\sum_{n=0}^{\infty} C_{n}^{(\mu)}(x, y ; \alpha, A) \xi^{n}=\left(\alpha I-x \xi \sqrt{2 A}+y \xi^{2} I\right)^{-\mu} \quad(\mu \neq 0),
\end{gathered}
$$

respectively.

We note the following special cases:

$$
\begin{gathered}
C_{n}^{(\mu)}(x, 1 ; 1, A)=C_{n}^{(\mu)}(x, A), \quad C_{n}^{(\mu)}(x, 1 ; 1,2)=C_{n}^{(\mu)}(x), \\
C_{n}^{(1)}(x, y ; 1, A)=U_{n}(x, y, A),
\end{gathered}
$$

where $U_{n}(x, y, A), C_{n}^{(\mu)}(x)$, and $C_{n}^{(\mu)}(x, A)$ denote the gCMaP defined by (5), the GeP defined by (3), and the GeMaP defined by (12), respectively.

\section{Generalized Gegenbauer Matrix Polynomials}

We use the 2I2VHMaP $H_{n, m}(x, y, A)$ (Table 1(II)) to introduce the 2-variable 1-parameter generalized Gegenbauer matrix polynomials (2V1PgGeMaP) ${ }_{(m)} C_{n}^{(\mu)}(x, y ; \alpha, A)$ in the following form:

$$
\begin{aligned}
& { }_{(m)} C_{n}^{(\mu)}(x, y ; \alpha, A) \\
& =\frac{1}{n ! \Gamma(\mu)} \int_{0}^{\infty} e^{-\alpha t} t^{n+\mu-1} H_{n, m}\left(x, \frac{y}{t^{m-1}}, A\right) d t .
\end{aligned}
$$

It is evident that in view of the relation that

$$
t^{n} H_{n, m}(x, y, A)=H_{n, m}\left(x t, y t^{m}, A\right) .
$$

Equation (20) can be expressed equivalently as

$$
{ }_{(m)} C_{n}^{(\mu)}(x, y ; \alpha, A)=\frac{1}{n ! \Gamma(\mu)} \int_{0}^{\infty} e^{-\alpha t} t^{\mu-1} H_{n, m}(x t, y t, A) d t .
$$

Now, making use of the series definition of $H_{n, m}(x, y, A)$ (Table 1(II)) and formula (11), we find that the 2V1PgGeMaP ${ }_{(m)} C_{n}^{(\mu)}(x, y ; \alpha, A)$ are defined by the following series:

$$
\begin{aligned}
{ }_{(m)} C_{n}^{(\mu)}(x, y ; \alpha, A) \\
=\frac{1}{\Gamma(\mu)} \sum_{k=0}^{[n / m]} \frac{(-y)^{k} \Gamma(n-(m-1) k+\mu)(x \sqrt{m A})^{n-m k}}{(n-m k) ! k ! \alpha^{n-(m-1) k+\mu}} .
\end{aligned}
$$

It is indeed easy to note the following special cases:

$$
\begin{gathered}
{ }_{(m)} C_{0}^{(\mu)}(x, y ; \alpha, A)=\alpha^{-\mu} I, \\
{ }_{(m)} C_{1}^{(\mu)}(x, y ; \alpha, A)=\frac{(x \sqrt{m A}) \mu}{\alpha^{\mu+1}}, \\
{ }_{(m)} C_{n}^{(\mu)}(x, 0 ; \alpha, A)=\frac{(x \sqrt{m A})^{n}(\mu)_{n}}{\alpha^{n+\mu} n !}, \\
{ }_{(2)} C_{n}^{(\mu)}(x, 1 ; 1, A)=C_{n}^{(\mu)}(x, A), \\
{ }_{(2)} C_{n}^{(\mu)}(x, y ; \alpha, A)=C_{n}^{(\mu)}(x, y ; \alpha, A), \\
C_{n}^{(\mu)}(x, y ; \alpha, A)=\frac{y^{n / 2}}{\alpha^{n+\mu-k}} C_{n}^{(\mu)}\left(\frac{x}{\sqrt{y}}, A\right), \\
{ }_{(2)} C_{n}^{(1)}(x, y ; 1, A)=U_{n}(x, y, A),
\end{gathered}
$$

where $(\mu)_{n}=\Gamma(\mu+n) / \Gamma(\mu)$ is the Pochhammer symbol.

The generating function for ${ }_{(m)} C_{n}^{(\mu)}(x, y ; \alpha, A)$ can be obtained with the help of the generating function of 2I2VHMaP (Table 1(II)). Multiplying (22) by $\xi^{n}$ and then summing up over $n$, we find

$$
\begin{aligned}
& \sum_{n=0}^{\infty}{ }_{(m)} C_{n}^{(\mu)}(x, y ; \alpha, A) \xi^{n} \\
& \quad=\frac{1}{\Gamma(\mu)} \int_{0}^{\infty} e^{-\alpha t} t^{\mu-1} \sum_{n=0}^{\infty} H_{n, m}(x t, y t, A) \frac{\xi^{n}}{n !} d t .
\end{aligned}
$$

Further, using generating function of $2 \mathrm{I} 2 \mathrm{VHMaP}$ in the r.h.s. of the above equation and then using relation (11) on the resultant equation, we get the following generating function for the 2V1PgGeMaP ${ }_{(m)} C_{n}^{(\mu)}(x, y ; \alpha, A)$ :

$$
\begin{array}{r}
\sum_{n=0}^{\infty}{ }_{(m)} C_{n}^{(\mu)}(x, y ; \alpha, A) \xi^{n}=\left(\alpha I-x \xi \sqrt{m A}+y \xi^{m} I\right)^{-\mu} \\
(\mu \neq 0) .
\end{array}
$$

Now, we can establish the generalization of ${ }_{(m)} C_{n}^{(\mu)}(x, y$; $\alpha, A$ ) by introducing the $3 \mathrm{~V} 1 \mathrm{PgGeMaP}{ }_{(m, s)} C_{n}^{(\mu)}(x, y, z ; \alpha, A)$ using 3I3VHMaP $H_{n}^{(m, s)}(x, y, z, A)$ (Table 1(III)) in the following form:

$$
\begin{aligned}
& { }_{(m, s)} C_{n}^{(\mu)}(x, y, z ; \alpha, A) \\
& \quad=\frac{1}{n ! \Gamma(\mu)} \int_{0}^{\infty} e^{-\alpha t} t^{n+\mu-1} H_{n}^{(m, s)}\left(x, \frac{y}{t^{m-1}}, \frac{z}{t^{s-1}}, A\right) d t .
\end{aligned}
$$

It is evident that in view of the relation that

$$
t^{n} H_{n}^{(m, s)}(x, y, z, A)=H_{n}^{(m, s)}\left(x t, y t^{m}, z t^{s}, A\right) .
$$


Equation (27) can be expressed equivalently as

$$
\begin{aligned}
{ }_{(m, s)} & C_{n}^{(\mu)}(x, y, z ; \alpha, A) \\
= & \frac{1}{n ! \Gamma(\mu)} \int_{0}^{\infty} e^{-\alpha t} t^{\mu-1} H_{n}^{(m, s)}(x t, y t, z t, A) d t .
\end{aligned}
$$

Further, proceeding on the same line as discussed above, we can obtain the following series definition and generating function for the $3 \mathrm{~V} 1 \mathrm{PgGeMaP}{ }_{(m, s)} C_{n}^{(\mu)}(x, y, z ; \alpha, A)$ :

$$
\begin{gathered}
{ }_{(m, s)} C_{n}^{(\mu)}(x, y, z ; \alpha, A) \\
=\frac{1}{\Gamma(\mu)} \sum_{k=0}^{[n / s]} \sum_{r=0}^{[(n-s k) / m]} \frac{(-1)^{r} \Gamma(n-(m-1) r-(s-1) k+\mu)}{(n-s k-m r) ! k ! r ! \alpha^{n-(m-1) r-(s-1) k+\mu}} \\
\times(-y)^{r} z^{k}(x \sqrt{m A})^{n-s k-m r},
\end{gathered}
$$

$$
\begin{aligned}
& \sum_{n=0}^{\infty}{ }_{(m, s)} C_{n}^{(\mu)}(x, y, z ; \alpha, A) \xi^{n} \\
& \quad=\left(\alpha I-x \xi \sqrt{m A}+y \xi^{m} I-z \xi^{s} I\right)^{-\mu} \quad(\mu \neq 0),
\end{aligned}
$$

respectively.

We note the following special cases:

$$
\begin{gathered}
{ }_{(m, s)} C_{0}^{(\mu)}(x, y, z ; \alpha, A)=\alpha^{-\mu} I, \\
{ }_{(m, s)} C_{1}^{(\mu)}(x, y, z ; \alpha, A)=\frac{(x \sqrt{m A}) \mu}{\alpha^{\mu+1}}, \\
{ }_{(m, s)} C_{n}^{(\mu)}(x, y, 0 ; \alpha, A)={ }_{m} C_{n}^{(\mu)}(x, y ; \alpha, A), \\
{ }_{(2, s)} C_{n}^{(1)}(x, y, 0 ; 1, A)=U_{n}(x, y, A) .
\end{gathered}
$$

Furthermore, using the integral representation (29), we establish some matrix differential recurrence relations for the $3 \mathrm{~V} 1 \mathrm{PgGeMaP}{ }_{(m, s)} C_{n}^{(\mu)}(x, y, z ; \alpha, A)$ with the help of the corresponding properties of the $3 \mathrm{I} 3 \mathrm{VHMaP} H_{n}^{(m, s)}(x, y, z, A)$. For example, the recurrence relations satisfied by the 3I3VHMaP $H_{n}^{(m, s)}(x, y, z, A)$ are derived in [8]. Now, replacing $x$ by $x t, y$ by $y t$, and $z$ by $z t$ in the equations $[8$, p. 228 (3.11), $229(3.14,3.15,3.20)]$ and using relation

$$
\frac{\partial}{\partial(t x)}=\frac{1}{t} \frac{\partial}{\partial x}
$$

we get the new set of recurrence relation. Again, multiplying the resultant equations by $e^{-\alpha t} t^{\nu-1} / n ! \Gamma(\nu)$, integrating it with respect to $t$ between the limits 0 to $\infty$, and then using the integral representation (29), we get the following recurrence relations for ${ }_{(m, s)} C_{n}^{(\mu)}(x, y, z ; \alpha, A)$ :

$$
\frac{\partial}{\partial x}(m, s)_{n} C_{n}^{(\mu)}(x, y, z ; \alpha, A)=\mu \sqrt{m A}_{(m, s)} C_{n-1}^{(\mu+1)}(x, y, z ; \alpha, A)
$$

$$
\begin{aligned}
& \frac{\partial^{m}}{\partial x^{m}(m, s)} C_{n}^{(\mu)}(x, y, z ; \alpha, A) \\
& =(\mu)_{m}(\sqrt{m A})_{(m, s)}^{m} C_{n-m}^{(\mu+m)}(x, y, z ; \alpha, A) \quad(n \geq m) \\
& \frac{\partial^{s}}{\partial x^{s}}(m, s) C_{n}^{(\mu)}(x, y, z ; \alpha, A) \\
& =(\mu)_{s}(\sqrt{m A})^{s}{ }_{(m, s)} C_{n-s}^{(\mu+s)}(x, y, z ; \alpha, A) \quad(n \geq s), \\
& x \frac{\partial}{\partial x}{ }_{(m, s)} C_{n}^{(\mu)}(x, y, z ; \alpha, A) \\
& -m y(\sqrt{m A})^{-1} \frac{\partial}{\partial x}{ }_{(m, s)} C_{n-m+1}^{(\mu)}(x, y, z ; \alpha, A) \\
& -s z(\sqrt{m A})^{-1} \frac{\partial}{\partial x}(m, s) C_{n-s+1}^{(\mu)}(x, y, z ; \alpha, A) \\
& =n_{(m, s)} C_{n}^{(\mu)}(x, y, z ; \alpha, A) \text {, }
\end{aligned}
$$

respectively.

Similarly, we can obtain other sets of recurrence relations for $3 \mathrm{~V} 1 \mathrm{PgGeMaP}{ }_{(m, s)} C_{n}^{(\mu)}(x, y, z ; \alpha, A)$ with the help of the corresponding properties of the 3I3VHMaP $H_{n}^{(m, s)}(x, y, z, A)$ as

$$
\begin{gathered}
\frac{\partial}{\partial y}{ }_{(m, s)} C_{n}^{(\mu)}(x, y, z ; \alpha, A)=-\mu_{(m, s)} C_{n-m}^{(\mu+1)}(x, y, z ; \alpha, A) \\
\frac{\partial}{\partial z}{ }_{(m, s)} C_{n}^{(\mu)}(x, y, z ; \alpha, A)=\mu_{(m, s)} C_{n-s}^{(\mu+1)}(x, y, z ; \alpha, A) \\
\frac{\partial^{r}}{\partial y^{r}(m, s)} C_{n}^{(\mu)}(x, y, z ; \alpha, A) \\
=(-1)^{r}(\mu)_{r(m, s)} C_{n-m r}^{(\mu+r)}(x, y, z ; \alpha, A), \\
\frac{\partial^{r}}{\partial z^{r}(m, s)} C_{n}^{(\mu)}(x, y, z ; \alpha, A)=(\mu)_{r(m, s)} C_{n-s r}^{(\mu+r)}(x, y, z ; \alpha, A) .
\end{gathered}
$$

Also, from the above relations we easily obtain

$$
\begin{aligned}
& \frac{\partial^{r}}{\partial x^{r}}(m, s) C_{n}^{(\mu)}(x, y, z ; \alpha, A) \\
& \quad+(-1)^{r-1}(\sqrt{m A})^{r} \frac{\partial^{r}}{\partial y^{r}(m, s)} C_{n+(m-1) r}^{(\mu)}(x, y, z ; \alpha, A)=0,
\end{aligned}
$$




$$
\begin{aligned}
& \frac{\partial^{r}}{\partial x^{r}(m, s)} C_{n}^{(\mu)}(x, y, z ; \alpha, A) \\
& \quad+(-1)^{-1}(\sqrt{m A})^{r} \frac{\partial^{r}}{\partial z^{r}}(m, s) C_{n+(s-1) r}^{(\mu+r)}(x, y, z ; \alpha, A)=0 .
\end{aligned}
$$

\section{Expansion of 3V1PgGeMaP}

In this section, we obtain the expansion of the $3 \mathrm{~V} 1 \mathrm{PgGeMaP}$ ${ }_{(m, s)} C_{n}^{(\mu)}(x, y, z ; \alpha, A)$ in the series of 3I3VHMaP $H_{n}^{(m, s)}(x, y$, $z, A)$. In order to obtain this, we first derive the expansion of $(x \sqrt{m A})^{n} I$ in the series of $H_{n}^{(m, s)}(x, y, z, A)$ by using the generating function (Table $1($ III)) in the form

$$
\begin{aligned}
(x \sqrt{m A})^{n} I= & n ! \sum_{k=0}^{[n / s]} \sum_{r=0}^{[(n-s k) / m]} \frac{(-1)^{k} y^{r} z^{k}}{k ! r !(n-s k-m r) !} \\
& \times H_{n-s k-m r}^{(m, s)}(x, y, z, A) \quad(n \geq 0) .
\end{aligned}
$$

Now, since

$$
\begin{aligned}
& { }_{(m, s)} C_{n}^{(\mu)}(x, y, z ; \alpha, A) \\
& =\frac{1}{\Gamma(\mu)} \sum_{k=0}^{[n / s][(n-s k) / m]} \sum_{r=0}^{(-1)^{r} \Gamma(n-(m-1) r-(s-1) k+\mu)} \\
& \times(-y)^{r} z^{k}(x \sqrt{m A})^{n-s k-m r},
\end{aligned}
$$

which on multiplying by $t^{n}$, using relation (see [12])

$$
\sum_{k=0}^{\infty} \sum_{r=0}^{k / m} A(r, k)=\sum_{k=0}^{\infty} \sum_{r=0}^{\infty} A(r, k+m r),
$$

and then using expression (37) in the resultant equation yield

$$
\begin{aligned}
& \sum_{n=0}^{\infty}(m, s) C_{n}^{(\mu)}(x, y, z ; \alpha, A) t^{n} \\
& =\frac{1}{\Gamma(\mu)} \sum_{n, k, r=0}^{\infty} \sum_{p=0}^{[n / s][(n-s k) / m]} \sum_{q=0}^{(n-s p-m q) ! k ! r ! p ! q ! \alpha^{n+r+k+\mu}} \\
& \quad \times y^{r+q} z^{k+p} H_{n-s p-m q}^{(m, s)}(x, y, z, A) t^{n+s k+m r} .
\end{aligned}
$$

Again, using relation (39) and in view of the fact

$$
\frac{(-1)^{k}}{(n-k) !}=\frac{(-n)_{k}}{n !}
$$

equation (40) becomes

$$
\begin{aligned}
\sum_{n=0}^{\infty}{ }_{(m, s)} C_{n}^{(\mu)}(x, y, z ; \alpha, A) t^{n} & \\
=\sum_{n=0}^{\infty} \sum_{k=0}^{n n / s][(n-s k) / m]} \sum_{r=0} \sum_{p=0}^{k} \sum_{q=0}^{r}( & \left((-1)^{r}(-k)_{p}(-r)_{q}\right. \\
& \left.\times(\mu)_{n-(s-1) k-(m-1) r}\right) \\
& \times((n-s k-m r) ! k ! r ! p ! q ! \\
& \left.\left.\times \alpha^{n-(s-1) k-(m-1) r+\mu}\right)^{-1}\right) \\
\times & ((\mu+n-(s-1) k \\
& \left.-(m-1) r)_{(s-1) p-(m-1) q}\right) \\
\times & \left.\left(\alpha^{(s-1) p-(m-1) q}\right)^{-1}\right) \\
\times & y^{r} z^{k} H_{n-s k-m r}^{(m, s)}(x, y, z, A) t^{n},
\end{aligned}
$$

which on equating coefficients of $t^{n}$ on both sides gives

$$
\begin{aligned}
& { }_{(m, s)} C_{n}^{(\mu)}(x, y, z ; \alpha, A) \\
& =\sum_{n=0}^{\infty} \sum_{k=0}^{[n / s][(n-s k) / m]} \sum_{r=0}^{(n-s k-m r) ! k ! r \alpha^{n-(s-1) k-(m-1) r+\mu}} \frac{(-1)^{r}(\mu)_{n-(s-1) k-(m-1) r}}{(n-s)} \\
& \times y^{r} z^{k} \Lambda_{k, r, n}^{(\mu)} H_{n-s k-m r}^{(m, s)}(x, y, z, A) t^{n},
\end{aligned}
$$

where

$$
\begin{aligned}
& \Lambda_{k, r, n}^{(\mu)} \\
& :=\sum_{p=0}^{k} \sum_{q=0}^{r} \frac{(-k)_{p}(-r)_{q}(\mu+n-(s-1) k-(m-1) r)_{(s-1) p-(m-1) q}}{p ! q ! \alpha^{(s-1) p-(m-1) q}} .
\end{aligned}
$$

\section{Bilinear and Bilateral Generating Matrix$$
\text { Relations for }{ }_{(m, s)} C_{n}^{(\mu)}(x, y, z ; \alpha, A)
$$

In order to derive several families of bilinear and bilateral generating matrix relations for the $3 \mathrm{~V} 1 \mathrm{PgGeMaP}$ ${ }_{(m, s)} C_{n}^{(\mu)}(x, y, z ; \alpha, A)$, we first state our result as follows.

Theorem 1. Corresponding to nonvanishing functions $\Omega_{\mu}\left(q_{1}, \ldots, q_{r}\right)$ consisting of $r$ (real or complex) variables $q_{1}, \ldots, q_{r}(r \in \mathbb{N})$ and of complex order $v$, let

$$
\Lambda_{\nu, \eta}\left(q_{1}, \ldots, q_{r} ; \psi\right):=\sum_{k=0}^{\infty} a_{k} \Omega_{\nu+\eta k}\left(q_{1}, \ldots, q_{r}\right) \psi^{k}
$$


and for $\left(a_{k} \neq 0, k \in \mathbb{N}_{0}, \eta \in \mathbb{C}\right)$,

$$
\begin{aligned}
& \Theta_{n, p, v, \eta}\left(x, y, z, \alpha ; q_{1}, \ldots, q_{r} ; \xi\right) \\
& :=\sum_{k=0}^{n / p} a_{k} C_{(m, s)}^{(\mu)} C_{n-p k}(x, y, z ; \alpha, A) \Omega_{\nu+\eta k}\left(q_{1}, \ldots, q_{r}\right) \xi^{k},
\end{aligned}
$$

where $n, p \in \mathbb{N}$ and $A$ is a matrix in $\mathbb{C}^{N \times N}$ satisfying the condition (1). Then one has

$$
\begin{gathered}
\sum_{n=0}^{\infty} \Theta_{n, p, v, \eta}\left(x, y, z, \alpha ; q_{1}, \ldots, q_{r} ; \frac{\gamma}{t^{p}}\right) t^{n} \\
=\left(\alpha I-x t \sqrt{m A}+y t^{m} I-z t^{s} I\right)^{-\mu} \\
\quad \times \Lambda_{\nu, \eta}\left(q_{1}, \ldots, q_{r} ; \gamma\right) \quad(\mu \neq 0),
\end{gathered}
$$

provided that each member of (47) exists.

Proof. Denote, for convenience, the first member of the assertion (47) of Theorem 1 by $S$. Then, upon substituting for the polynomial $\Theta_{n, p, v, \eta}\left(x, y, z, \alpha ; q_{1}, \ldots, q_{r} ; \xi\right)$ which comes from (46) into the left hand side of (47), we get

$$
\begin{aligned}
S=\sum_{n=0}^{\infty} \sum_{k=0}^{n / p} a_{k}{ }_{(m, s)} C_{n-p k}^{(\mu)}(x, y, z ; \alpha, A) \Omega_{\nu+\eta k}\left(q_{1}, \ldots, q_{r}\right) \\
\quad \times \gamma^{k} t^{n-p k} .
\end{aligned}
$$

Now replacing $n$ by $n+p k$ in the r.h.s. of (48) and using relation (39) in the resultant equation, we find

$$
\begin{aligned}
S & =\sum_{n=0}^{\infty} \sum_{k=0}^{\infty} a_{k(m, s)} C_{n}^{(\mu)}(x, y, z ; \alpha, A) \Omega_{\nu+\eta k}\left(q_{1}, \ldots, q_{r}\right) \gamma^{k} t^{n} \\
& =\sum_{n=0}^{\infty}(m, s) C_{n}^{(\mu)}(x, y, z ; \alpha, A) t^{n} \sum_{k=0}^{\infty} a_{k} \Omega_{\nu+\eta k}\left(q_{1}, \ldots, q_{r}\right) \gamma^{k} \\
& =\left(\alpha I-x t \sqrt{m A}+y t^{m} I-z t^{s} I\right)^{-\mu} \Lambda_{\nu, \eta}\left(q_{1}, \ldots, q_{r} ; \gamma\right),
\end{aligned}
$$

which proves the assertion (47) of Theorem 1.

In order to discuss further applications of Theorem of 1 , we consider the multivariable function $\Omega_{\nu+\eta k}\left(q_{1}, \ldots, q_{r}\right)(k \in$ $\mathbb{N}_{0}, r \in \mathbb{N}$ ) in terms of the functions of one or more variables. For example, consider the case of $r=2$ and $\Omega_{\nu+\eta k}(u, v)=L_{\nu+\eta k}^{(B, \lambda)}(u, v)$ in Theorem 1, where $L_{\nu+\eta k}^{(B, \lambda)}(u, v)$ denotes the 2VLMaP (Table $1(\mathrm{IV})$ ). Then we obtain the following result which provides a class of bilateral generating matrix relations for $3 \mathrm{~V} 1 \mathrm{PgGeMaP}{ }_{(m, s)} C_{n}^{(\mu)}(x, y, z ; \alpha, A)$ and $2 \operatorname{VLMaP} L_{n}^{(A, \lambda)}(x, y)$.

Corollary 2. Let

$$
\Lambda_{\nu, \eta}(u, v ; \psi):=\sum_{k=0}^{\infty} a_{k} L_{\nu+\eta k}^{(B, \lambda)}(u, v) \psi^{k},
$$

and for $\left(a_{k} \neq 0, v, \eta \in \mathbb{N}_{0}\right)$,

$$
\begin{aligned}
& \Theta_{n, p, v, \eta}(x, y, z, \alpha ; u, v ; \xi) \\
& \quad:=\sum_{k=0}^{n / p} a_{k} C_{(m, s)} C_{n-p k}^{(\mu)}(x, y, z ; \alpha, A) L_{\nu+\eta k}^{(B, \lambda)}(u, v) \xi^{k},
\end{aligned}
$$

where $n, p \in \mathbb{N}$, and $A$ and $B$ is a matrix in $\mathbb{C}^{N \times N}$ satisfying the condition (1) and $B+n I$ invertible for every integer $n>0$. Then we have

$$
\begin{aligned}
& \sum_{n=0}^{\infty} \Theta_{n, p, v, \eta}\left(x, y, z, \alpha ; u, v ; \frac{\gamma}{t^{p}}\right) t^{n} \\
& \quad=\left(\alpha I-x t \sqrt{m A}+y t^{m} I-z t^{s} I\right)^{-\mu} \Lambda_{v, \eta}(u, v ; \gamma) \quad(\mu \neq 0),
\end{aligned}
$$

provided that each member of (52) exists.

Remark 3. Using the generating matrix functions for $2 \operatorname{VLMaP} L_{k}^{(B, \lambda)}(u, v)$ (Table $\left.1(\mathrm{IV})\right)$ and taking $a_{k}=1=\eta$ and $\nu=0$ in the generating matrix relations (52), we get

$$
\begin{aligned}
& \sum_{n=0}^{\infty} \sum_{k=0}^{n / p}{ }_{(m, s)} C_{n-p k}^{(\mu)}(x, y, z ; \alpha, A) L_{k}^{(B, \lambda)}(u, v) \gamma^{k} t^{n-p k} \\
& =\left(\alpha I-x t \sqrt{m A}+y t^{m} I-z t^{s} I\right)^{-\mu} \times(1-\gamma v)^{-(B+I)} \\
& \quad \times \exp \left(\frac{-\lambda u \gamma}{1-\gamma v}\right) \quad(|\gamma v|<1, \mu \neq 0),
\end{aligned}
$$

which for $v=1$ gives

$$
\begin{aligned}
& \sum_{n=0}^{\infty} \sum_{k=0}^{n / p}{ }_{(m, s)} C_{n-p k}^{(\mu)}(x, y, z ; \alpha, A) L_{k}^{(B, \lambda)}(u) \gamma^{k} t^{n-p k} \\
& =\left(\alpha I-x t \sqrt{m A}+y t^{m} I-z t^{s} I\right)^{-\mu} \times(1-\gamma)^{-(B+I)} \\
& \quad \times \exp \left(\frac{-\lambda u \gamma}{1-\gamma}\right) \quad(|\gamma|<1, \mu \neq 0)
\end{aligned}
$$

Also, taking $z=0$ and using relation (32b) in (53), we find

$$
\begin{gathered}
\sum_{n=0}^{\infty} \sum_{k=0}^{n / p}{ }_{(m)} C_{n-p k}^{(\mu)}(x, y ; \alpha, A) L_{k}^{(B, \lambda)}(u, v) \gamma^{k} t^{n-p k} \\
=\left(\alpha I-x t \sqrt{m A}+y t^{m} I\right)^{-\mu} \times(1-\gamma v)^{-(B+I)} \\
\quad \times \exp \left(\frac{-\lambda u \gamma}{1-\gamma v}\right) \quad(|\gamma v|<1, \mu \neq 0),
\end{gathered}
$$

where ${ }_{(p)} C_{k}^{(\rho)}(u, v ; \beta, B)$ denotes the 2 V1PgGeMaP defined by (25) and for $v=1,(55)$ reduces to the bilateral generating matrix relations for $2 \mathrm{~V} 1 \mathrm{PgGeMaP}{ }_{(m)} C_{n}^{(\mu)}(x, y ; \alpha, A)$ and $\operatorname{LMaP} L_{n}^{(A, \lambda)}(x)$. 
Further taking $\alpha=1=\mu, m=2, z=0$ and using relation (32c) in (53), we find

$$
\begin{aligned}
\sum_{n=0}^{\infty} \sum_{k=0}^{n / p} U_{n-p k}(x, y, A) L_{k}^{(B, \lambda)}(u, v) \gamma^{k} t^{n-p k} \\
=\left(I-x t \sqrt{2 A}+y t^{2} I\right)^{-1} \times(1-\gamma v)^{-(B+I)} \\
\quad \times \exp \left(\frac{-\lambda u \gamma}{1-\gamma v}\right) \quad(|\gamma v|<1),
\end{aligned}
$$

which for $y=v=1$ gives a known bilateral generating matrix relations $[13$, p.30].

Again, set $r=2$ and $\Omega_{\nu+\eta k}(u, v)=f_{v+\eta k}(u, v, B)$ in Theorem 1, where $f_{v+\eta k}(u, v, B)$ denotes the 2VMLMaP (Table $1(\mathrm{~V})$ ). Then, we obtain the following result which provides a class of bilateral generating matrix relations for $3 \mathrm{~V} 1 \mathrm{PgGeMaP}{ }_{(m, s)} C_{n}^{(\mu)}(x, y, z ; \alpha, A)$ and 2VMLMaP $f_{n}^{(A, \lambda)}(x, y)$.

\section{Corollary 4. Let}

$$
\Lambda_{\nu, \eta}(u, v ; \psi):=\sum_{k=0}^{\infty} a_{k} f_{v+\eta k}^{(B, \lambda)}(u, v) \psi^{k},
$$

and for $\left(a_{k} \neq 0, v, \eta \in \mathbb{N}_{0}\right)$,

$$
\begin{aligned}
& \Theta_{n, p, v, \eta}(x, y, z, \alpha ; u, v ; \xi) \\
& \quad:=\sum_{k=0}^{n / p} a_{k}{ }_{(m, s)} C_{n-p k}^{(\mu)}(x, y, z ; \alpha, A) f_{\nu+\eta k}^{(B, \lambda)}(u, v) \xi^{k},
\end{aligned}
$$

where $n, p \in \mathbb{N}$, and $A$ and $B$ is a matrix in $\mathbb{C}^{N \times N}$ satisfying the condition (1). Then we have

$$
\begin{aligned}
& \sum_{n=0}^{\infty} \Theta_{n, p, v, \eta}\left(x, y, z, \alpha ; u, v ; \frac{\gamma}{t^{p}}\right) t^{n} \\
& \quad=\left(\alpha I-x t \sqrt{m A}+y t^{m} I-z t^{s} I\right)^{-\mu} \Lambda_{\nu, \eta}(u, v ; \gamma) \quad(\mu \neq 0),
\end{aligned}
$$

provided that each member of (59) exists.

Remark 5. Using the generating matrix functions for 2VMLMaP $f_{k}^{(B, \lambda)}(u, v)($ Table $1(\mathrm{~V}))$ and taking $a_{k}=1=\eta$ and $v=0$ in the generating matrix relations (59), we get

$$
\begin{aligned}
\sum_{n=0}^{\infty} \sum_{k=0}^{n / p}{ }_{(m, s)} C_{n-p k}^{(\mu)}(x, y, z ; \alpha, A) f_{k}^{(B, \lambda)}(u, v) \gamma^{k} t^{n-p k} \\
=\left(\alpha I-x t \sqrt{m A}+y t^{m} I-z t^{s} I\right)^{-\mu} \times(1-\gamma v)^{-B} \\
\quad \times \exp (\lambda u \gamma) \quad(\mu \neq 0),
\end{aligned}
$$

which for $v=1$ gives

$$
\begin{aligned}
\sum_{n=0}^{\infty} \sum_{k=0}^{n / p}{ }_{(m, s)} C_{n-p k}^{(\mu)}(x, y, z ; \alpha, A) f_{k}^{(B, \lambda)}(u) \gamma^{k} t^{n-p k} \\
=\left(\alpha I-x t \sqrt{m A}+y t^{m} I-z t^{s} I\right)^{-\mu} \times(1-\gamma)^{-B} \\
\quad \times \exp (\lambda u \gamma) \quad(\mu \neq 0) .
\end{aligned}
$$
find

Also, taking $z=0$ and using relation (32b) in (60), we

$$
\begin{aligned}
\sum_{n=0}^{\infty} \sum_{k=0}^{n / p}{ }_{(m)} C_{n-p k}^{(\mu)}(x, y ; \alpha, A) f_{k}^{(B, \lambda)}(u, v) \gamma^{k} t^{n-p k} \\
=\left(\alpha I-x t \sqrt{m A}+y t^{m} I\right)^{-\mu} \times(1-\gamma v)^{-B} \\
\quad \times \exp (\lambda u \gamma) \quad(\mu \neq 0),
\end{aligned}
$$

which for $v=1$ gives bilateral generating matrix relations for 2V1PgGeMaP ${ }_{(m)} C_{n}^{(\mu)}(x, y ; \alpha, A)$ and MLMaP $f_{n}^{(A, \lambda)}(x)$.

Further taking $\alpha=1=\mu, m=2, z=0$ and using relation $(32 c)$ in (60), we find

$$
\begin{aligned}
& \sum_{n=0}^{\infty} \sum_{k=0}^{n / p} U_{n-p k}(x, y, A) f_{k}^{(B, \lambda)}(u, v) \gamma^{k} t^{n-p k} \\
& \quad=\left(I-x t \sqrt{2 A}+y t^{2} I\right)^{-1}(1-\gamma v)^{-B} \exp (\lambda u \gamma) .
\end{aligned}
$$

Next, set $r=2$ and $\Omega_{\nu+\eta k}(u, v)=U_{\nu+\eta k}(u, v, B)$ in Theorem 1, where $U_{v+\eta k}(u, v, B)$ denotes the gCMaP defined by (5). Then, we obtain the following result which provides a class of bilateral generating matrix relations for $3 \mathrm{~V} 1 \mathrm{PgGeMaP}$ ${ }_{(m, s)} C_{n}^{(\mu)}(x, y, z ; \alpha, A)$ and $\mathrm{gCMaP} U_{n}(x, y, A)$.

Corollary 6. Let

$$
\Lambda_{\nu, \eta}(u, v ; \psi):=\sum_{k=0}^{\infty} a_{k} U_{v+\eta k}(u, v, B) \psi^{k}
$$

and for $\left(a_{k} \neq 0, v, \eta \in \mathbb{N}_{0}\right)$,

$$
\begin{aligned}
& \Theta_{n, p, v, \eta}(x, y, z, \alpha ; u, v ; \xi) \\
& :=\sum_{k=0}^{n / p} a_{k} C_{(m, s)} C_{n-p k}^{(\mu)}(x, y, z ; \alpha, A) U_{v+\eta k}(u, v, B) \xi^{k},
\end{aligned}
$$

where $n, p \in \mathbb{N}$, and $A$ and $B$ is a matrix in $\mathbb{C}^{N \times N}$ satisfying the condition (1). Then we have

$$
\begin{aligned}
& \sum_{n=0}^{\infty} \Theta_{n, p, v, \eta}\left(x, y, z, \alpha ; u, v ; \frac{\gamma}{t^{p}}\right) t^{n} \\
& \quad=\left(\alpha I-x t \sqrt{m A}+y t^{m} I-z t^{s} I\right)^{-\mu} \Lambda_{\nu, \eta}(u, v ; \gamma) \quad(\mu \neq 0),
\end{aligned}
$$

provided that each member of (66) exists.

Remark 7. Using the generating matrix functions (6) for $\operatorname{gCMaP} U_{k}(u, v, B)$ and taking $a_{k}=1=\eta$ and $v=0$ in the generating matrix relations (66), we get

$$
\begin{aligned}
& \sum_{n=0}^{\infty} \sum_{k=0}^{n / p}{ }_{(m, s)} C_{n-p k}^{(\mu)}(x, y, z ; \alpha, A) U_{k}(u, v, B) \gamma^{k} t^{n-p k} \\
&=\left(\alpha I-x t \sqrt{m A}+y t^{m} I-z t^{s} I\right)^{-\mu}\left(I-u \gamma \sqrt{2 B}+v \gamma^{2} I\right)^{-1} \\
& \quad\left(|B|<\frac{1}{\sqrt{2}},|\gamma|<1, \mu \neq 0\right),
\end{aligned}
$$


which for $v=1$ gives

$$
\begin{gathered}
\sum_{n=0}^{\infty} \sum_{k=0}^{n / p}{ }_{(m, s)} C_{n-p k}^{(\mu)}(x, y, z ; \alpha, A) U_{k}(u, B) \gamma^{k} t^{n-p k} \\
=\left(\alpha I-x t \sqrt{m A}+y t^{m} I-z t^{s} I\right)^{-\mu}\left(I-u \gamma \sqrt{2 B}+\gamma^{2} I\right)^{-1} \\
\quad\left(|B|<\frac{1}{\sqrt{2}},|\gamma|<1, \mu \neq 0\right) .
\end{gathered}
$$
find

Also, taking $z=0$ and using relation (32b) in (67), we

$$
\begin{aligned}
& \sum_{n=0}^{\infty} \sum_{k=0}^{n / p}{ }_{(m)} C_{n-p k}^{(\mu)}(x, y ; \alpha, A) U_{k}(u, v, B) \gamma^{k} t^{n-p k} \\
&=\left(\alpha I-x t \sqrt{m A}+y t^{m} I\right)^{-\mu}\left(I-u \gamma \sqrt{2 B}+v \gamma^{2} I\right)^{-1} \\
& \quad\left(|B|<\frac{1}{\sqrt{2}},|\gamma|<1, \mu \neq 0\right),
\end{aligned}
$$

which for $v=1$ gives bilateral generating matrix relations for 2V1PgGeMaP ${ }_{(m)} C_{n}^{(\mu)}(x, y ; \alpha, A)$ and $\mathrm{CMaP}_{n}(x, A)$.

Further, taking $\alpha=1=\mu, m=2$, and $z=0$ and using relation (32c) in (67), we find a bilinear generating matrix relations

$$
\begin{aligned}
& \sum_{n=0}^{\infty} \sum_{k=0}^{n / p} U_{n-p k}(x, y, A) U_{k}(u, v, B) \gamma^{k} t^{n-p k} \\
&=\left(I-x t \sqrt{2 A}+y t^{2} I\right)^{-1}\left(I-u \gamma \sqrt{2 B}+v \gamma^{2} I\right)^{-1} \\
&\left(|B|<\frac{1}{\sqrt{2}},|\gamma|<1\right)
\end{aligned}
$$

which for $y=v=1$ gives known bilinear generating matrix relations [13, p.31].

Again, set $r=2$ and $\Omega_{v+\eta k}(u, v)=H_{v+\eta k}(u, v, B)$ in Theorem 1, where $H_{\nu+\eta k}(u, v, B)$ denotes the 2VHMaP (Table $1(\mathrm{I})$ ). Then we obtain the following result which provides a class of bilateral generating matrix relations for $3 \mathrm{~V} 1 \mathrm{PgGeMaP}{ }_{(m, s)} C_{n}^{(\mu)}(x, y, z ; \alpha, A)$ and 2VHMaP $H_{n}(x, y, A)$.

\section{Corollary 8. Let}

$$
\Lambda_{\nu, \eta}(u, v ; \psi):=\sum_{k=0}^{\infty} a_{k} H_{\nu+\eta k}(u, v, B) \psi^{k}
$$

and for $\left(a_{k} \neq 0, v, \eta \in \mathbb{N}_{0}\right)$,

$$
\begin{aligned}
& \Theta_{n, p, v, \eta}(x, y, z, \alpha ; u, v ; \xi) \\
& :=\sum_{k=0}^{n / p} a_{k}{ }_{(m, s)} C_{n-p k}^{(\mu)}(x, y, z ; \alpha, A) H_{\nu+\eta k}(u, v, B) \xi^{k},
\end{aligned}
$$

where $n, p \in \mathbb{N}$, and $A$ and $B$ is a matrix in $\mathbb{C}^{N \times N}$ satisfying the condition (1). Then we have

$$
\begin{aligned}
& \qquad \begin{array}{l}
\sum_{n=0}^{\infty} \Theta_{n, p, v, \eta}\left(x, y, z, \alpha ; u, v ; \frac{\gamma}{t^{p}}\right) t^{n} \\
=\left(\alpha I-x t \sqrt{m A}+y t^{m} I-z t^{s} I\right)^{-\mu} \Lambda_{\nu, \eta}(u, v ; \gamma) \\
(\mu \neq 0),
\end{array} \\
& \text { provided that each member of }(73) \text { exists. }
\end{aligned}
$$

Remark 9. Using the generating matrix functions for $2 \mathrm{VHMaP} H_{k}(u, v, B)$ and taking $a_{k}=1 / k !, \eta=1$ and $v=0$ in the generating matrix relations (73), we get

$$
\begin{aligned}
\sum_{n=0}^{\infty} \sum_{k=0}^{n / p}{ }_{(m, s)} C_{n-p k}^{(\mu)}(x, y, z ; \alpha, A) H_{k}(u, v, B) \frac{\gamma^{k}}{k !} t^{n-p k} \\
=\left(\alpha I-x t \sqrt{m A}+y t^{m} I-z t^{s} I\right)^{-\mu} \exp \left(u \gamma \sqrt{2 B}-v \gamma^{2} I\right) \\
\quad\left(|B|<\frac{1}{\sqrt{2}},|\gamma|<1, \mu \neq 0\right),
\end{aligned}
$$

which for $v=1$ yields

$$
\begin{aligned}
& \sum_{k=0}^{\infty} \sum_{k=0}^{n / p}{ }_{(m, s)} C_{n-p k}^{(\mu)}(x, y, z ; \alpha, A) H_{k}(u, B) \gamma^{k} t^{n-p k} \\
& =\left(\alpha I-x t \sqrt{m A}+y t^{m} I-z t^{s} I\right)^{-\mu} \exp \left(u \gamma \sqrt{2 B}-\gamma^{2} I\right) \\
& \quad\left(|B|<\frac{1}{\sqrt{2}},|\gamma|<1, \mu \neq 0\right) .
\end{aligned}
$$

Also, taking $z=0$ and using relation (32b) in (74), we find

$$
\begin{aligned}
& \sum_{n=0}^{\infty} \sum_{k=0}^{n / p} C_{(m)}^{(\mu)}(x-p k(x, y ; \alpha, A) H_{k}(u, v, B) \frac{\gamma^{k}}{k !} t^{n-p k} \\
&=\left(\alpha I-x t \sqrt{m A}+y t^{m} I\right)^{-\mu} \exp \left(u \gamma \sqrt{2 B}-v \gamma^{2} I\right) \\
&\left(|B|<\frac{1}{\sqrt{2}},|\gamma|<1, \mu \neq 0\right),
\end{aligned}
$$

which for $v=1$ gives bilateral generating matrix relations for 2V1PgGeMaP ${ }_{(m)} C_{n}^{(\mu)}(x, y ; \alpha, A)$ and $\operatorname{HMaP}_{n}(x, A)$.

Further, taking $\alpha=1=\mu, m=2, z=0$ and using relation (32c) in (74), we obtain

$$
\begin{aligned}
\sum_{n=0}^{\infty} \sum_{k=0}^{n / p} U_{n-p k}(x, y, A) H_{k}(u, v, B) \frac{\gamma^{k}}{k !} t^{n-p k} \\
=\left(I-x t \sqrt{2 A}+y t^{2} I\right)^{-1} \exp \left(u \gamma \sqrt{2 B}-v \gamma^{2} I\right) \\
\quad\left(|B|<\frac{1}{\sqrt{2}},|\gamma|<1\right),
\end{aligned}
$$

which for $y=v=1$ gives a known bilateral generating matrix relations [13, p.32]. 
Again set $r=3$ and $\Omega_{\nu+\eta k}(u, v, w)={ }_{(p, q)} C_{\nu+\eta k}^{(\rho)}(u, v, w ; \beta$, $B$ ) in Theorem 1, where ${ }_{(p, q)} C_{\nu+\eta k}^{(\rho)}(u, v, w ; \beta, B)$ denotes the $3 \mathrm{~V} 1 \mathrm{PgGeMaP}$ defined by (30). Then we obtain the following result which provides a class of bilinear generating matrix relations for $3 \mathrm{~V} 1 \mathrm{PgGeMaP}{ }_{(m, s)} C_{n}^{(\mu)}(x, y, z ; \alpha, A)$.

Corollary 10. Let

$$
\Lambda_{v, \eta}(u, v, w ; \beta, \psi):=\sum_{k=0}^{\infty} a_{k}{ }_{(p, q)} C_{\nu+\eta k}^{(\rho)}(u, v, w ; \beta, B) \psi^{k}
$$

and for $\left(a_{k} \neq 0, v, \eta \in \mathbb{N}_{0}\right)$,

$$
\begin{aligned}
& \Theta_{n, p, v, \eta}(x, y, z, \alpha ; u, v, w, \beta ; \xi) \\
& :=\sum_{k=0}^{n / p} a_{k} C_{(m, s)} C_{n-p k}^{(\mu)}(x, y, z ; \alpha, A){ }_{(p, q)} C_{\nu+\eta k}^{(\rho)}(u, v, w ; \beta, B) \psi^{k},
\end{aligned}
$$

where $n, p \in \mathbb{N}$, and $A$ and $B$ is a matrix in $\mathbb{C}^{N \times N}$ satisfying the condition (1). Then we have

$$
\begin{aligned}
& \sum_{n=0}^{\infty} \Theta_{n, p, v, \eta}\left(x, y, z, \alpha ; u, v, w, \beta ; \frac{\gamma}{t^{p}}\right) t^{n} \\
& \quad=\left(\alpha I-x t \sqrt{m A}+y t^{m} I-z t^{s} I\right)^{-\mu} \Lambda_{\nu, \eta}(u, v, w, \beta ; \gamma),
\end{aligned}
$$

provided that each member of (80) exists.

Remark 11. Using the generating matrix functions (31) for 3V1PgGeMaP ${ }_{(m, s)} C_{n}^{(\mu)}(x, y, z ; \alpha, A)$ and taking $a_{k}=1=\eta$ and $v=0$ in the generating matrix relations (80), we get

$$
\begin{aligned}
\sum_{n=0}^{\infty} \sum_{k=0}^{n / p}{ }_{(m, s)} C_{n-p k}^{(\mu)}(x, y, z ; \alpha, A) & { }_{(p, q)} C_{k}^{(\rho)}(u, v, w ; \beta, B) \gamma^{k} t^{n-p k} \\
= & \left(\alpha I-x t \sqrt{m A}+y t^{m} I-z t^{s} I\right)^{-\mu} \\
& \times\left(\beta I-u \gamma \sqrt{p B}+v \gamma^{p} I-w \gamma^{q} I\right)^{-\rho} \quad(\mu, \rho \neq 0) .
\end{aligned}
$$

Further, taking $w=0$ and using relation (32b) in the previous equation, we find

$$
\begin{aligned}
\sum_{n=0}^{\infty} \sum_{k=0}^{n / p}{ }_{(m, s)} C_{n-p k}^{(\mu)}(x, y, z ; \alpha, A)_{(p)} C_{k}^{(\rho)}(u, v ; \beta, B) \gamma^{k} t^{n-p k} \\
=\left(\alpha I-x t \sqrt{m A}+y t^{m} I-z t^{s} I\right)^{-\mu} \\
\quad \times\left(\beta I-u \gamma \sqrt{p B}+v t^{p} I\right)^{-\rho} \quad(\mu, \rho \neq 0) .
\end{aligned}
$$

Also, taking $\alpha=1=\mu, m=2$, and $w=z=0$ and using relation ((32b), (32c)) in (81), we get

$$
\begin{array}{r}
\sum_{n=0}^{\infty} \sum_{k=0}^{n / p} U_{n-p k}(x, y, A){ }_{(p)} C_{k}^{(\rho)}(u, v ; \beta, B) \gamma^{k} t^{n-p k} \\
=\left(I-x t \sqrt{2 A}+y t^{2} I\right)^{-1}\left(\beta I-u \gamma \sqrt{p B}+v \gamma^{p} I\right)^{-\rho} \\
\left(|A|<\frac{1}{\sqrt{2}},|t|<1, \rho \neq 0\right) .
\end{array}
$$

\section{Concluding Remarks}

Very recently, Dattoli et al. [14] introduced the 2-variable generalized Legendre polynomials (2VgLeP) $P_{n}(x, y \backslash m)$, defined by the series

$$
\begin{aligned}
P_{n} & (x, y \backslash m) \\
& =\frac{1}{\sqrt{\pi}} \sum_{k=0}^{[n / m]} \frac{\Gamma(n+1 / 2-(m-1) k)(-x)^{n-m k}(-y)^{k}}{(n-m k) ! k !} .
\end{aligned}
$$

These polynomials are introduced by taking the action of the following operator [14, p.84 (3.4)]:

$$
\widehat{\Pi}^{(m)}(x, y):=\frac{1}{\sqrt{\pi}} \int_{0}^{\infty} e^{-t} t^{-1 / 2}(-t)^{x D_{x}} \exp \left(-t y \frac{\partial^{m}}{\partial x^{m}}\right) d t,
$$

on $x^{n} / n$ ! and then using the operational definition of 2variable Hermite-Kampé de Fériet polynomials (2VHKdFP) $H_{n}^{(m)}(x, y)[15]$

$$
H_{n}^{(m)}(x, y)=\exp \left(y \frac{\partial^{m}}{\partial x^{m}}\right)\{x\}^{n}
$$

and the property

$$
r^{x D_{x}} f(x)=f(r x),
$$

of the dilation operator [15], so as to obtain

$$
\begin{aligned}
P_{n}(x, y \backslash m) & =\widehat{\Pi}^{(m)}(x, y)\left\{\frac{x^{n}}{n !}\right\} \\
& =\frac{1}{n ! \sqrt{\pi}} \int_{0}^{\infty} e^{-t} t^{-1 / 2} H_{n}^{(m)}(-x t,-y t) d t,
\end{aligned}
$$

which yields definition (84).

In order to take the advantage of this technique, we introduce the following operator $\widehat{\Pi}_{\mu}(x, y ; \alpha, A)$ :

$$
\begin{aligned}
& \widehat{\Pi}_{\nu}(x, y ; \alpha, A) \\
& \quad:=\frac{1}{\Gamma(\mu)} \int_{0}^{\infty} e^{-\alpha t} t^{\mu-1}(\sqrt{2 A} t)^{x D_{x}} \exp \left(-t y \frac{\partial^{2}}{\partial x^{2}}\right) d t .
\end{aligned}
$$


Now, operating $\widehat{\Pi}_{\nu}(x, y ; \alpha, A)$ on $x^{n} / n$ ! and then making use of (86), (87), (9), and (15), we obtain the following operational representation for the $2 \mathrm{~V} 1 \mathrm{PGeMaP} C_{n}^{(\nu)}(x, y ; \alpha, A)$ :

$$
C_{n}^{(\nu)}(x, y ; \alpha, A)=\widehat{\Pi}_{\nu}(x, y ; \alpha, A)\left\{\frac{x^{n}}{n !}\right\},
$$

which for $\alpha=y=1$ and in view of relation (19) yields the following operational representation for the GeMaP $C_{n}^{(\nu)}(x, A)$ :

$$
C_{n}^{(v)}(x, A)=\widehat{\Pi}_{\nu}(x, A)\left\{\frac{x^{n}}{n !}\right\},
$$

where

$$
\begin{aligned}
\widehat{\Pi}_{\nu}(x, A) & =\widehat{\Pi}_{\nu}(x, 1 ; 1, A) \\
& =\frac{1}{\Gamma(\mu)} \int_{0}^{\infty} e^{-\alpha t} t^{\mu-1}(\sqrt{2 A} t)^{x D_{x}} \exp \left(-t \frac{\partial^{2}}{\partial x^{2}}\right) d t .
\end{aligned}
$$

Now, to find the operational representation for the 3V1PgGeMaP ${ }_{(m, s)} C_{n}^{(\mu)}(x, y, z ; \alpha, A)$, we introduce the following generalization of operator (89):

$$
\begin{aligned}
& { }_{(m, s)} \widehat{\Pi}_{\mu}(x, y, z ; \alpha, A) \\
& :=\frac{1}{\Gamma(\mu)} \int_{0}^{\infty} e^{-\alpha t} t^{\mu-1}(\sqrt{m A} t)^{x D_{x}} \\
& \quad \times \exp \left(-t y \frac{\partial^{m}}{\partial x^{m}}+t z \frac{\partial^{s}}{\partial x^{s}}\right) d t,
\end{aligned}
$$

which on using (27), (87), and the operational rule of 3I3VHMaP $H_{n}^{(m, s)}(x, y, z, A)[8]$

$$
\begin{aligned}
& H_{n}^{(m, s)}(x, y, z, A) \\
& \quad=\exp \left(z(\sqrt{m A})^{-s} \frac{\partial^{s}}{\partial x^{s}}-y(\sqrt{m A})^{-m} \frac{\partial^{m}}{\partial x^{m}}\right)\left\{(x \sqrt{m A})^{n}\right\}
\end{aligned}
$$

gives

$$
{ }_{(m, s)} C_{n}^{(\mu)}(x, y, z ; \alpha, A)=_{(m, s)} \widehat{\Pi}_{\mu}(x, y, z ; \alpha, A)\left\{\frac{x^{n}}{n !}\right\} .
$$

Taking $z=0$ in (95) and using relations ((32a), (32b), and $(32 \mathrm{c})$ ), we find the following operational representation for the 2V1PgGeMaP ${ }_{(m)} C_{n}^{(\mu)}(x, y ; \alpha, A)$ :

$$
{ }_{(m)} C_{n}^{(\mu)}(x, y ; \alpha, A)={ }_{(m)} \widehat{\Pi}_{\mu}(x, y ; \alpha, A)\left\{\frac{x^{n}}{n !}\right\},
$$

where

$$
\begin{aligned}
{ }_{(m)} & \widehat{\Pi}_{\mu}(x, y ; \alpha, A) \\
= & { }_{(m, s)} \widehat{\Pi}_{\mu}(x, y, 0 ; \alpha, A) \\
= & \frac{1}{\Gamma(\mu)} \int_{0}^{\infty} e^{-\alpha t} t^{\mu-1}(\sqrt{m A} t)^{x D_{x}} \exp \left(-t y \frac{\partial^{m}}{\partial x^{m}}\right) d t .
\end{aligned}
$$

In particular, we note that

$$
{ }_{(2, s)} \widehat{\Pi}_{\mu}(x, y, 0 ; \alpha, A)=\widehat{\Pi}_{\mu}(x, y ; \alpha, A) .
$$

In this paper, several new matrix polynomials are introduced using integral transform method allowing the derivation of a wealth of relations involving these polynomials. These results allow us to note that the use of the method of the integral representation is a fairly important tool of analysis and can be usefully extended to other families of polynomials which is a problem for further research.

\section{Conflict of Interests}

The author declares that there is no conflict of interests regarding the publication of this paper.

\section{Acknowledgment}

The author is thankful to the referees for several useful comments and suggestions towards the improvement of this paper.

\section{References}

[1] L. Jódar, R. Company, and E. Ponsoda, "Orthogonal matrix polynomials and systems of second order differential equations," Differential Equations and Dynamical Systems, vol. 3, no. 3, pp. 269-288, 1995.

[2] R. S. Batahan, "A new extension of Hermite matrix polynomials and its applications," Linear Algebra and Its Applications, vol. 419, no. 1, pp. 82-92, 2006.

[3] E. Defez, M. Garcia-Honrubia, and R. J. Villanueva, "A procedure for computing the exponential of a matrix using Hermite matrix polynomials," Far East Journal of Applied Mathematics, vol. 6, no. 3, pp. 217-231, 2002.

[4] E. Defez and L. Jódar, "Some applications of the Hermite matrix polynomials series expansions," Journal of Computational and Applied Mathematics, vol. 99, no. 1-2, pp. 105-117, 1998.

[5] L. Jódar and R. Company, "Hermite matrix polynomials and second order matrix differential equations," Approximation Theory and its Applications, vol. 12, no. 2, pp. 20-30, 1996.

[6] L. Jódar, R. Company, and E. Navarro, "Laguerre matrix polynomials and systems of second-order differential equations," Applied Numerical Mathematics, vol. 15, no. 1, pp. 53-63, 1994.

[7] M. S. Metwally, M. T. Mohamed, and A. Shehata, "Generalizations of two-index two-variable Hermite matrix polynomials," Demonstratio Mathematica, vol. 42, no. 4, pp. 687-701, 2009.

[8] S. Khan and A. A. Al-Gonah, "Multi-variable Hermite matrix polynomials: properties and applications," Journal of Mathematical Analysis and Applications, vol. 412, no. 1, pp. 222-235, 2014.

[9] S. Khan and N. A. M. Hassan, "Variable Laguerre matrix polynomials and Lie-algebraic techniques," Journal of Physics A: Mathematical and Theoretical, vol. 43, no. 23, Article ID 235204, pp. 1-21, 2010.

[10] L. C. Andrews, Special Functions for Engineers and Applied Mathematicians, Macmillan, New York, NY, USA, 1985.

[11] G. Dattoli, S. Lorenzutta, and C. Cesarano, "From Hermite to Humbert polynomials," Rendiconti dell'Istituto di Matematica dell'Università di Trieste, vol. 35, no. 1-2, pp. 37-48, 2003. 
[12] H. M. Srivastava and H. L. Manocha, A Treatise on Generating Functions, Halsted Press, New York, NY, USA, 1984.

[13] A. Altin and B. Çekim, "Generating matrix functions for Chebyshev matrix polynomials of the second kind," Hacettepe Journal of Mathematics and Statistics, vol. 41, no. 1, pp. 25-32, 2012.

[14] G. Dattoli, B. Germano, M. R. Martinelli, and P. E. Ricci, "A novel theory of Legendre polynomials," Mathematical and Computer Modelling, vol. 54, no. 1-2, pp. 80-87, 2011.

[15] G. Dattoli, P. L. Ottaviani, A. Torre, and L. Vázquez, "Evolution operator equations: integration with algebraic and finitedifference methods. Applications to physical problems in classical and quantum mechanics and quantum field theory," Rivista del Nuovo Cimento della Società Italiana di Fisica, vol. 20, no. 2, pp. 1-133, 1997. 


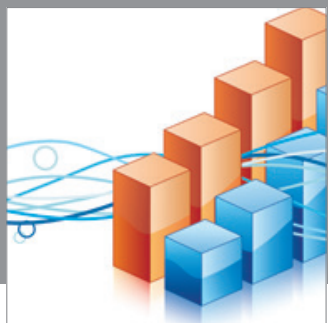

Advances in

Operations Research

mansans

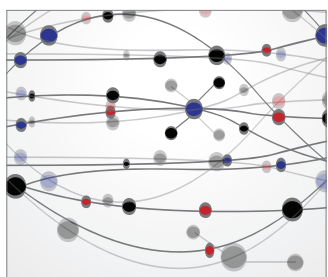

The Scientific World Journal
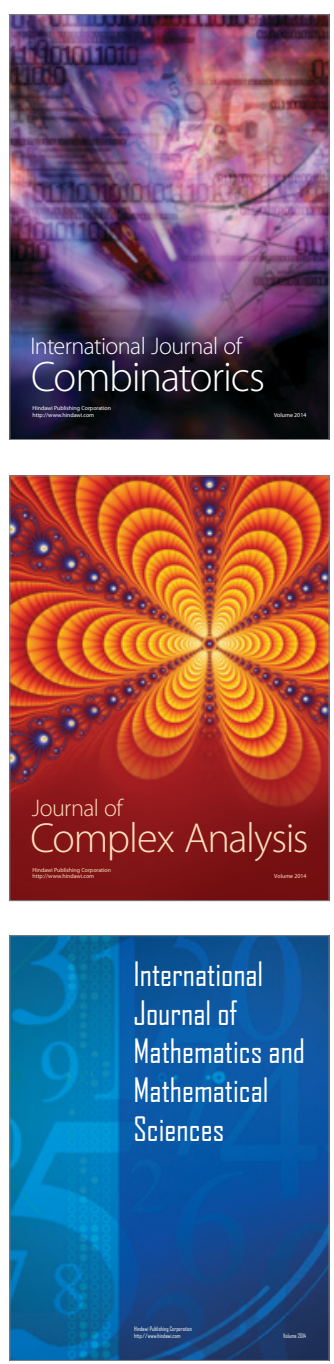
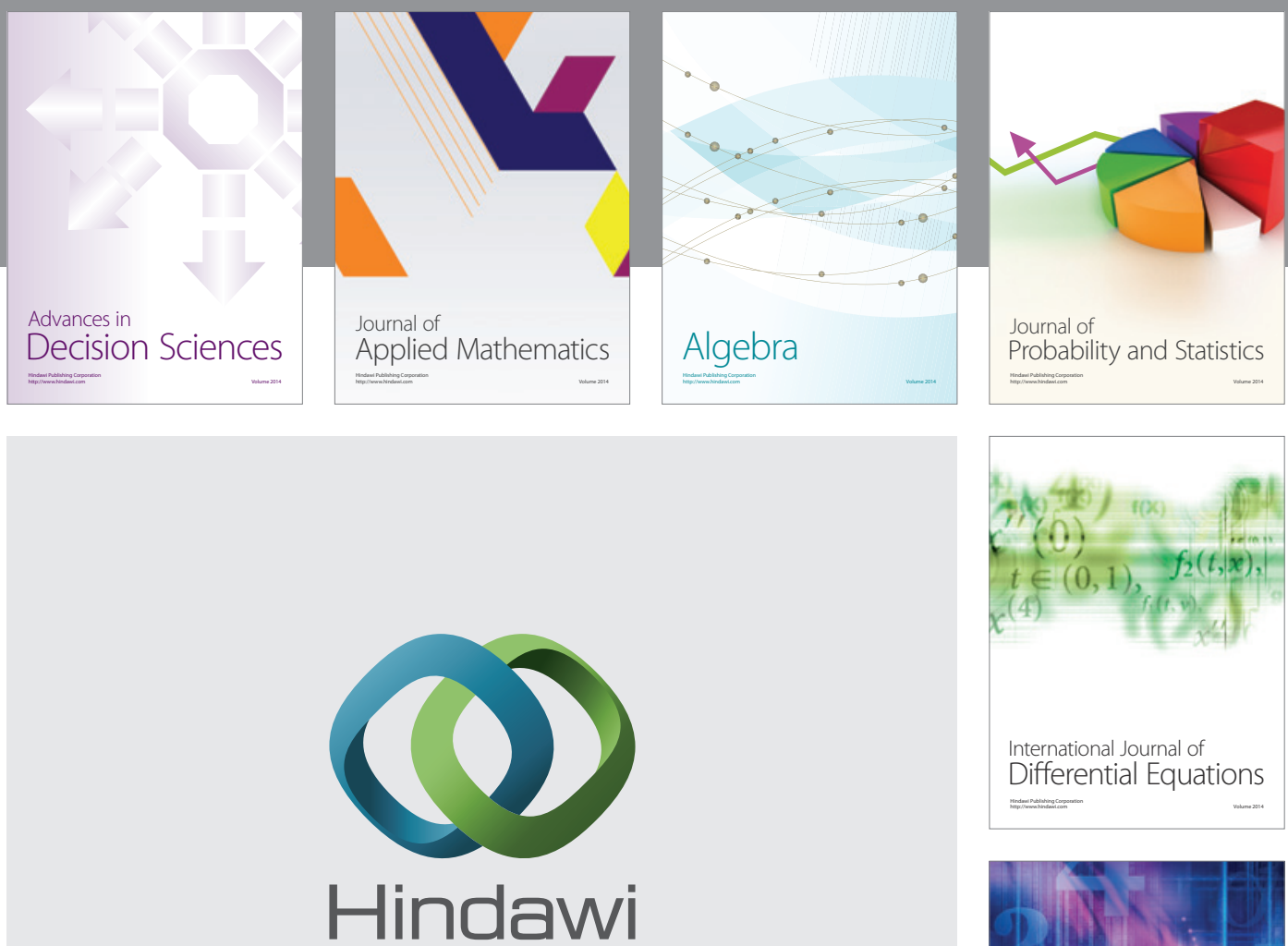

Submit your manuscripts at http://www.hindawi.com
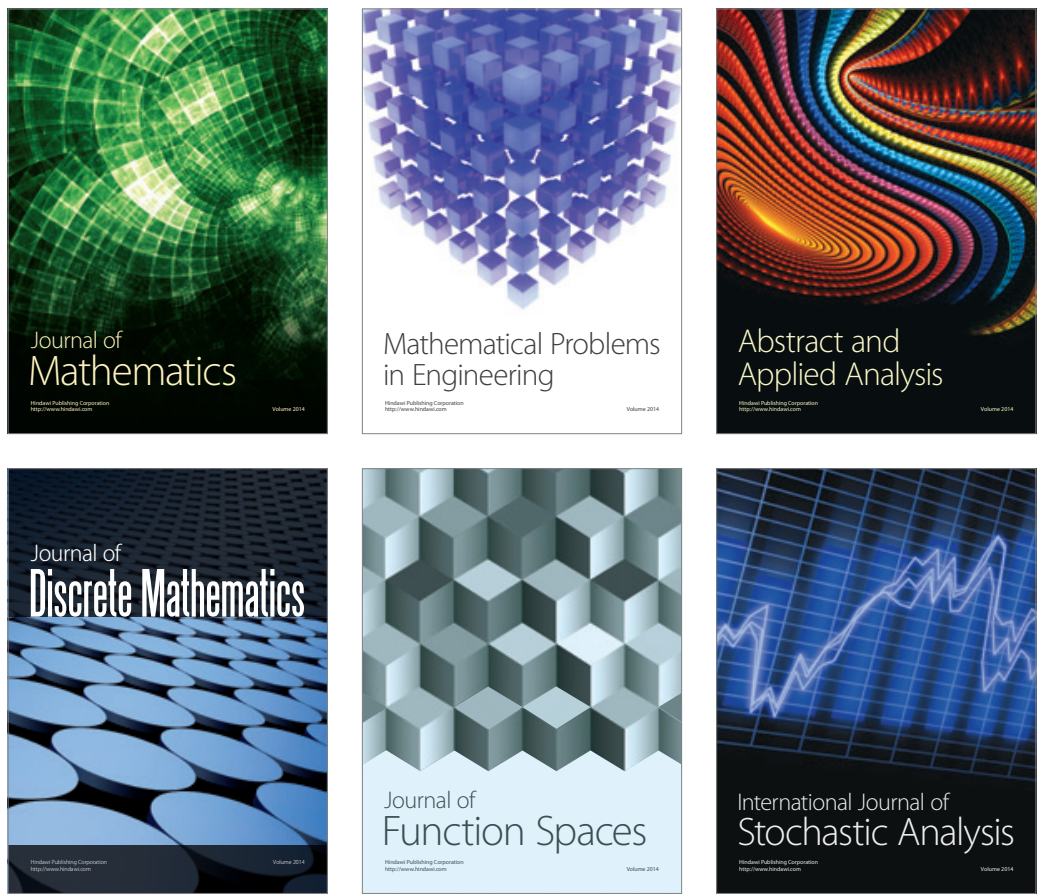

Journal of

Function Spaces




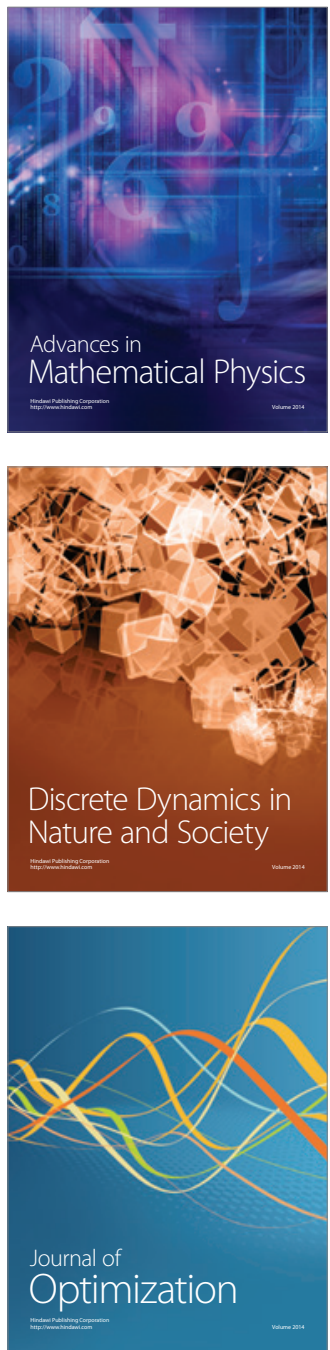